\title{
Cold Neutron Energy Dependent Production of Ultracold Neutrons in Solid Deuterium
}

\author{
F. Atchison, ${ }^{1}$ B. Blau, ${ }^{1}$ K. Bodek, ${ }^{2}$ B. van den Brandt, ${ }^{1}$ T. Bryś,,${ }^{1,}$ M. Daum, ${ }^{1}$ P. Fierlinger, ${ }^{1, \dagger}$ A. Frei, ${ }^{3}$ P. Geltenbort, ${ }^{4}$ \\ P. Hautle, ${ }^{1}$ R. Henneck, ${ }^{1}$ S. Heule, ${ }^{1,}$ A. Holley, ${ }^{5}$ M. Kasprzak, ${ }^{1, \S}$ K. Kirch, ${ }^{1, \|}$ A. Knecht, ${ }^{1,}$ J. A. Konter, ${ }^{1}$ M. Kuźniak, ${ }^{2, \rrbracket}$ \\ C.-Y. Liu, ${ }^{6}$ C. L. Morris, ${ }^{7}$ A. Pichlmaier, ${ }^{1}$ C. Plonka, ${ }^{4}$ Y. Pokotilovski, ${ }^{8}$ A. Saunders, ${ }^{7}$ Y. Shin, ${ }^{6}$ D. Tortorella, ${ }^{3}$ \\ M. Wohlmuther, ${ }^{1}$ A. R. Young, ${ }^{5}$ J. Zejma, ${ }^{2}$ and G. Zsigmond ${ }^{1}$ \\ ${ }^{1}$ Paul Scherrer Institut (PSI), CH-5232 Villigen PSI, Switzerland \\ ${ }^{2}$ Institute of Physics, Jagiellonian University, 30-059 Cracow, Poland \\ ${ }^{3}$ Technische Universität München, D-85748 Garching, Germany \\ ${ }^{4}$ Institut Laue-Langevin (ILL), 38042 Grenoble Cedex 9, France \\ ${ }^{5}$ North Carolina State University, Raleigh, North Carolina 27695, USA \\ ${ }^{6}$ Indiana University, Bloomington, Indiana 47408, USA \\ ${ }^{7}$ Los Alamos National Laboratory, Los Alamos, New Mexico 87545, USA \\ ${ }^{8}$ Joint Institute for Nuclear Research, Dubna, Moscow Region, 141980, Russia
}

(Received 29 June 2007; published 27 December 2007)

\begin{abstract}
A measurement of the production of ultracold neutrons from velocity-selected cold neutrons on gaseous and solid deuterium targets is reported. The expected energy dependence for two-particle collisions with well defined neutron and Maxwell-Boltzmann distributed molecular velocities is found for the gas target. The solid target data agree in shape with the phonon density-of-states curve and provide strong evidence for the phonon model including multiphonon excitations.
\end{abstract}

DOI: 10.1103/PhysRevLett.99.262502

The strong interaction of slow neutrons with matter can be described in a neutron optics picture using a material dependent index of refraction [1-3] or using material potentials that are easily calculated from the nuclear density and the coherent scattering lengths via the Fermi pseudopotential [4]. Most materials exhibit repulsive wall potentials, e.g., $\mathrm{Al}(\sim 50 \mathrm{neV}), \mathrm{Be}$ and $\mathrm{Ni}(\sim 250 \mathrm{neV})$, diamond $(\sim 300 \mathrm{neV})$, and solid deuterium $\left({ }^{2} \mathrm{H}_{2}\right.$, $\sim 100 \mathrm{neV}$ ). Because of lower densities, potentials of gases are usually much smaller, e.g., $\sim 1 \mathrm{neV}$ for gaseous ${ }^{2} \mathrm{H}_{2}$ at atmospheric pressure and $25 \mathrm{~K}$. Neutrons with kinetic energies below such material potentials can be trapped in vacuum inside matter vessels. These neutrons are referred to as ultracold neutrons (UCN) and are used in fundamental physics experiments, such as the search for a neutron electric dipole moment or measurements of the neutron lifetime. Stored UCN behave similarly to a gas with milli-Kelvin temperatures (in mechanical equilibrium) but with a strong influence from gravity $(\sim 100 \mathrm{neV} / \mathrm{m})$. For reviews on $\mathrm{UCN}$ physics the reader is referred to $[5,6]$.

Most UCN experiments require considerably more intense sources than are available today. Present efforts for improvements [7] follow two main directions: UCN production in either superfluid helium [8] or solid ${ }^{2} \mathrm{H}_{2}$. The potential of solid ${ }^{2} \mathrm{H}_{2}$ as a good cold neutron $(\mathrm{CN})$ moderator and UCN converter was recognized more than 20 years ago [9-11] and has been demonstrated in test experiments over the past years [12-14]. A suitable ${ }^{2} \mathrm{H}_{2}$ UCN converter has low temperature to avoid thermal upscattering and high ortho ${ }^{2} \mathrm{H}_{2}$ concentration $\left(c_{o}\right)$ in order to avoid upscattering on para ${ }^{2} \mathrm{H}_{2}$ [15]. Recently, the relevant slow neutron cross sections of gaseous, liquid
PACS numbers: 28.20.Cz, 29.25.Dz, 61.12.Ex

[16], and solid ${ }^{2} \mathrm{H}_{2}$ [17] have been measured, as well as the integral UCN production cross section from a cold neutron beam [18].

Only in the case of ${ }^{2} \mathrm{H}_{2}$ gas is there a rigorous solution for the double differential cross section by Young and Koppel (Y-K) $[19,20]$. Solid ${ }^{2} \mathrm{H}_{2}$ requires a rather different approach because of the material structure. For solid ${ }^{2} \mathrm{H}_{2}$, the temperature dependence of UCN lifetimes [13], the total scattering [17] and UCN production cross sections [18] were well described by a simple theoretical model $[10,11]$ in which one calculates the downscattering of cold neutrons via phonon creation in solid ${ }^{2} \mathrm{H}_{2}$; see [21-23]. Including multiphonon excitations in the calculation improves the agreement with the measured ${ }^{2} \mathrm{H}_{2}$ temperature dependence of the UCN production [18]. Although the agreement between experiment and calculation is remarkably good, the phonon model's ability at predicting the double differential cross sections, particularly in the regions specific to UCN production, has not really been tested.

The downscattering of $\mathrm{CN}$ into $\mathrm{UCN}$ via phonon creation in solid deuterium and using the incoherent approximation is calculated following [22]; compare also [24]:

$$
\begin{aligned}
\sigma\left(E_{\mathrm{CN}} \rightarrow E_{\mathrm{UCN}}\right)= & \sigma_{0} \sqrt{\frac{E_{\mathrm{UCN}}}{E_{\mathrm{CN}}}} \exp \left[-\frac{E_{\mathrm{CN}}+E_{\mathrm{UCN}}}{\mu \tau}\right] \\
& \times \sum_{n=1}^{\infty} \frac{g_{n}\left(E_{\mathrm{CN}}-E_{\mathrm{UCN}}\right)}{n !}\left(\frac{E_{\mathrm{CN}}+E_{\mathrm{UCN}}}{\mu}\right)^{n} .
\end{aligned}
$$

Because $E_{\mathrm{UCN}} \ll E_{\mathrm{CN}}$ one can use $\varepsilon \approx E_{\mathrm{CN}}-E_{\mathrm{UCN}} \approx$ $E_{\mathrm{CN}}+E_{\mathrm{UCN}} \approx E_{\mathrm{CN}}$ in Eq. (1). We use the character- 
istic bound scattering cross section $\sigma_{0}=4 \pi\left(a_{\text {coh }}^{2}+\right.$ $\left.a_{\text {inc }}^{2}\right)=7.63$ barn and the mass ratio of deuteron to neutron $\mu=\frac{m_{d}}{m_{n}}$. Sometimes $\tau=\frac{\hbar^{2}}{2 m_{d} \gamma}$ is referred to as the "characteristic energy." The quantity $\gamma=\frac{\hbar^{2}}{2 m_{d}} \int_{0}^{\infty} \frac{1}{\varepsilon} \times$ $\operatorname{coth}\left(\frac{\varepsilon}{2 k_{B} T}\right) g(\varepsilon) d \varepsilon$ is the average of the square displacement of ${ }^{2} \mathrm{H}_{2}$ in the lattice over the normalized phonon density of states $g(\varepsilon)$. With $g_{1}(\varepsilon) \equiv g(\varepsilon)$, the multiphonon contributions are calculated via $g_{n}(\varepsilon)=\int_{-\infty}^{\infty} g_{n-1}\left(\varepsilon^{\prime}\right) g(\varepsilon-$ $\left.\varepsilon^{\prime}\right) d \varepsilon^{\prime}$. The density of states can be represented by either a simple Debye spectrum or a more realistic phonon spectrum [11,25]. For the Debye model, $g(\varepsilon)=\frac{3 \varepsilon^{2}}{\varepsilon_{D}^{3}}$ [for $0 \leq$ $\varepsilon \leq \varepsilon_{D}$ and otherwise $g(\varepsilon)=0$ ] with $\varepsilon_{D}=k_{B} T_{D}$ and the Debye temperature $T_{D} \approx 110 \mathrm{~K}$ of solid ${ }^{2} \mathrm{H}_{2}$ [26]. In order to compare with measurements, Eq. (1) is integrated over the incident $\mathrm{CN}$ spectrum and the relevant UCN spectrum [27].

In order to improve understanding of the underlying processes, we have measured the UCN production from gaseous and solid ${ }^{2} \mathrm{H}_{2}$ targets using a velocity-selected $\mathrm{CN}$ beam. The experiment was performed on the $\mathrm{CN}$ beam line for fundamental physics (FUNSPIN) [28] at the Swiss spallation neutron source SINQ. A schematic diagram of the experimental setup is displayed in Fig. 1. The cryogenic and UCN parts were similar to the ones used in [18], using the same ${ }^{2} \mathrm{H}_{2}$ gas system and cryogenic target (see also [29]), the same UCN detector, and the UCN guide system with only minor modifications. The relevant changes in the setup as compared to [18] were the use of a velocity selector (VS) [30] working in air in front of the target cryostat and a time-of-flight (TOF) system for $\mathrm{CN}$ behind the first UCN mirror. Depending on the specific settings of the VS (rotation frequency and tilt angle), velocity resolutions of about $20 \%$, measured with neutron TOF (1\%-5\% resolution), were achieved. The corresponding energy spectra for the selected bins are displayed in

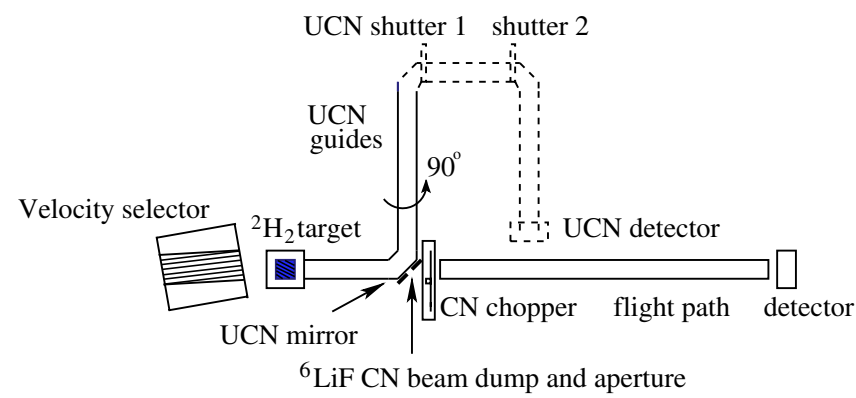

FIG. 1 (color online). The schematical setup (not to scale): The $\mathrm{CN}$ beam passes through the velocity selector (VS) before hitting the ${ }^{2} \mathrm{H}_{2}$ target. Behind the target, UCN are reflected upwards by a mirror that is transparent for $\mathrm{CN}$. The major part of the $\mathrm{CN}$ beam is dumped behind the mirror while the central fraction passes to the time-of-flight detection system. The UCN are simultaneously guided away from the $\mathrm{CN}$ beam axis to a well shielded UCN detector (compare [18]).
Fig. 2. After passing a $38 \mathrm{~mm}$ aperture and a $125 \mu \mathrm{m} \mathrm{Zr}$ vacuum window, $\mathrm{CN}$ from the FUNSPIN beam entered the $40 \mathrm{~mm}$ long ${ }^{2} \mathrm{H}_{2}$ target. Downstream of the target, a UCN guide system (Ni coated stainless steel and Be coated glass) was mounted in which after roughly $0.6 \mathrm{~m}$ a first mirror separated UCN (and some very cold neutrons; see [18]) from the $\mathrm{CN}$ beam by reflecting them upwards. The UCN were again reflected by $90^{\circ}$ by a second mirror at about $1 \mathrm{~m}$ height into a horizontal guide section perpendicular to the axis of the incident beam; this helped to further filter the UCN spectrum [27] and reduced beam induced background in the detector system. UCN passed the $1.4 \mathrm{~m}$ long horizontal section and fell by about $1 \mathrm{~m}$ into a well shielded ${ }^{3} \mathrm{He}$ gas detector. In order to reach the TOF system, CN passed through the UCN mirror $(100 \mu \mathrm{m} \mathrm{Al}$ with diamondlike carbon coating), a $10 \mathrm{~mm}$ hole in the attached ${ }^{6} \mathrm{LiF}$ beam dump, and a $100 \mu \mathrm{m} \mathrm{Al}$ vacuum exit window. The TOF system had a total flight path of $2830 \mathrm{~mm}$ and consisted of a one-disk-one-slit chopper (operated in air at $25 \mathrm{~Hz}$ and about 1/300 open to close ratio), a flight tube flushed with He to reduce neutron losses (2.5 m length, $16 \mu \mathrm{m} \mathrm{Al}$ entrance and exit windows), and a "thin" CN detector (the same as used in [28]). "Thin" refers to a small amount of ${ }^{3} \mathrm{He}$ in the counting gas, which should result in a $1 / v$ dependence of the detector efficiency on CN velocity $v$. The TOF system was used to monitor the $\mathrm{CN}$ beam intensity and spectrum. It also allowed measurement of the energy dependent $\mathrm{CN}$ attenuation for various target conditions from which one can

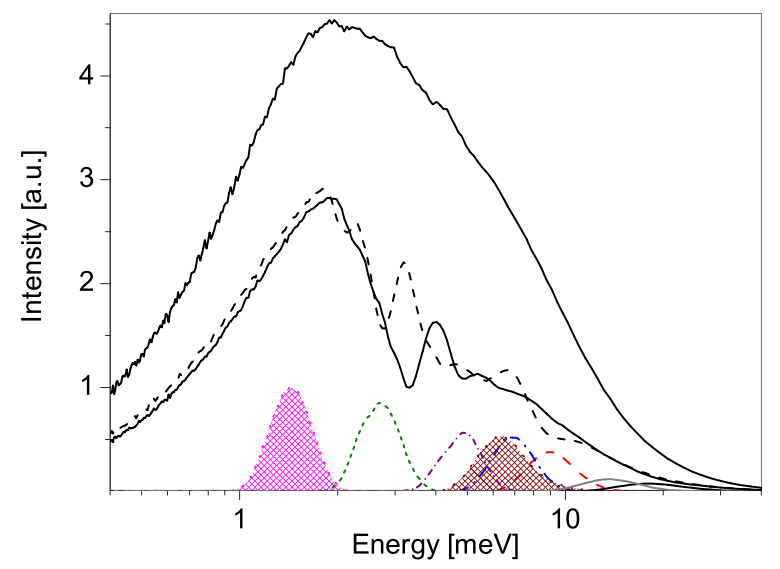

FIG. 2 (color online). Detector efficiency and proton beam charge corrected $\mathrm{CN}$ spectra at FUNSPIN. The three full range spectra were measured without velocity selector (VS) and show the transmission of the empty target (solid black line) and for two different ${ }^{2} \mathrm{H}_{2}$ crystals at $8 \mathrm{~K}$; the Bragg cutoff is at $2 \mathrm{meV}$; transmissions differ due to different crystallite orientations. The other energy distributions were obtained for various VS settings and normalized as the other spectra. The $\mathrm{CN}$ flux was measured with $\mathrm{Au}$ foil activation and is $(4.6 \pm 0.5) \times 10^{7} \mathrm{~cm}^{-2} \mathrm{~mA}^{-1} \mathrm{~s}^{-1}$ for the full spectrum. For the shaded spectra at $1.4 \mathrm{meV}$ and $6.3 \mathrm{meV}$ the measured flux is $(1.7 \pm 0.2) \times 10^{6} \mathrm{~cm}^{-2} \mathrm{~mA}^{-1} \mathrm{~s}^{-1}$ and $(4.4 \pm 0.4) \times 10^{6} \mathrm{~cm}^{-2} \mathrm{~mA}^{-1} \mathrm{~s}^{-1}$, respectively. 
calculate total scattering cross sections. Figure 2 shows CN spectra (without VS and normalized to the same proton charge) for the empty target cell and for two different ${ }^{2} \mathrm{H}_{2}$ crystals, frozen from the liquid phase and cooled to $8 \mathrm{~K}$.

While the $\mathrm{CN}$ detector continuously monitored relative changes in the $\mathrm{CN}$ flux, an absolute calibration of the flux was performed using standard gold foil activation in front of the target for a given proton beam charge on SINQ. The gold foils, of diameter $20 \mathrm{~mm}$, were centered on the beam axis. The gold activity was measured on a calibrated $\mathrm{HpGe}$ $\gamma$ detector system a few days later. Because of scheduling issues the activation time had to be rather short, resulting in low activities and about $10 \%$ uncertainties. The expected $1 / v$ dependence for detector efficiency was confirmed by comparing, for the same VS settings and empty target cell, gold foil activation and count rates in the $\mathrm{CN}$ detector. The homogenous illumination of the target at different VS settings has been checked by measuring $\mathrm{CN}$ beam profiles over the accepted beam spot by $\mathrm{Cu}$ foil activation and subsequent readout with imaging plates.

Having calibrated the $\mathrm{CN}$ detector, the next aim is to normalize the $\mathrm{UCN}$ production to the $\mathrm{CN}$ flux. Because of the good agreement between the recent measurement [18] and the theoretical expectation [11] for the energy integrated production cross section, one can here allow for an overall scale factor (different for gas and solid) for UCN transport and detection efficiencies. This is straightforward for the gas target: $\mathrm{CN}$ interactions in the low density target are homogenously distributed along the axis and, thus, the UCN extraction from the target is independent of $\mathrm{CN}$ energy and only statistical uncertainties apply for the gas cross sections.

The situation is more complex for solid ${ }^{2} \mathrm{H}_{2}$; the shape of the UCN energy spectrum at the instant of production does not depend on $\mathrm{CN}$ energy, but the spatial distribution of $\mathrm{CN}$ interactions and, thus, of the UCN production does; this leads to a $\mathrm{CN}$ energy dependent UCN extraction efficiency. Relative energy dependent corrections have been determined by modeling the UCN extraction from the target cell, starting the UCN according to calculated axial $\mathrm{CN}$ flux distributions, which are consistent with the measured $\mathrm{CN}$ transmissions (the average of the two "without VS" results, shown in Fig. 2). The variation of the measured transmissions for different crystals causes the dominant uncertainty in the modeling, and makes a significant contribution to the cross section error. The relative energy dependent corrections are included in Fig. 2. The overall, absolute efficiency for extracting, transporting, and detecting produced UCN is of the order of 0.003 for the setup [18]; however, it does not affect the present analysis: we here use calculated cross sections for normalization and compare the shape of the $\mathrm{CN}$ energy dependence of measured data and theoretical calculation (see below).

The typical background at the UCN detection system without proton beam to SINQ was less than $0.003 \mathrm{~s}^{-1}$.
With proton beam, and the $\mathrm{CN}$ beam on the ${ }^{2} \mathrm{H}_{2}$ target, the background rate was $(0.0054 \pm 0.0003) \mathrm{s}^{-1}$, as measured with one or both UCN shutters closed. Within statistics, this rate was independent of VS settings and not affected by small variations in the proton beam current as this was very stable during data taking, $(1252 \pm 3) \mu \mathrm{A}$. Typical UCN count rates with UCN shutters open were in the region 0.01 to $0.06 \mathrm{~s}^{-1}$.

UCN production cross sections were extracted from the measured UCN rates (corrected for background) and normalized to the $\mathrm{CN}$ flux as corrected for extraction efficiency. A final normalization was performed in which a constant factor that minimized the mean square deviation between the cross section model and the measured results was applied to the data. The cross section values are shown in Fig. 3 for gaseous ortho ${ }^{2} \mathrm{H}_{2}\left(c_{o} \approx 0.98\right.$, prepared and analyzed as in [29]) at $25 \mathrm{~K}$ and a pressure of $0.12 \mathrm{MPa}$ and in Fig. 4 for solid ortho ${ }^{2} \mathrm{H}_{2}$ at $8 \mathrm{~K}$ (in equilibrium with its saturated vapor pressure in the gas system above the target). For each data point the normalized velocity-selected $\mathrm{CN}$ spectrum is displayed on the $\mathrm{CN}$ energy axis. Along with the data, the model calculations are shown as a continuous function of $\mathrm{CN}$ energy (black line) and averaged over the velocity-selected $\mathrm{CN}$ spectra (red squares), respectively.

For the gas measurement the fit of the 8 data points to the Y-K model results in $\chi^{2}=8.54$ for 7 degrees of freedom $\left(\chi_{\text {red }}^{2}=1.22\right)$, confirming the proper understanding of the UCN production in gaseous ${ }^{2} \mathrm{H}_{2}$, a reasonable control of systematic effects in the experiment, and the statistical origin of deviations between model and data. Qualitatively, the energy dependence is easy to understand: The $\mathrm{CN}$ require ${ }^{2} \mathrm{H}_{2}$ molecules of a certain velocity class in the direction of the $\mathrm{CN}$ beam for the downscattering. As the Maxwell-Boltzmann distribution peaks at zero for any

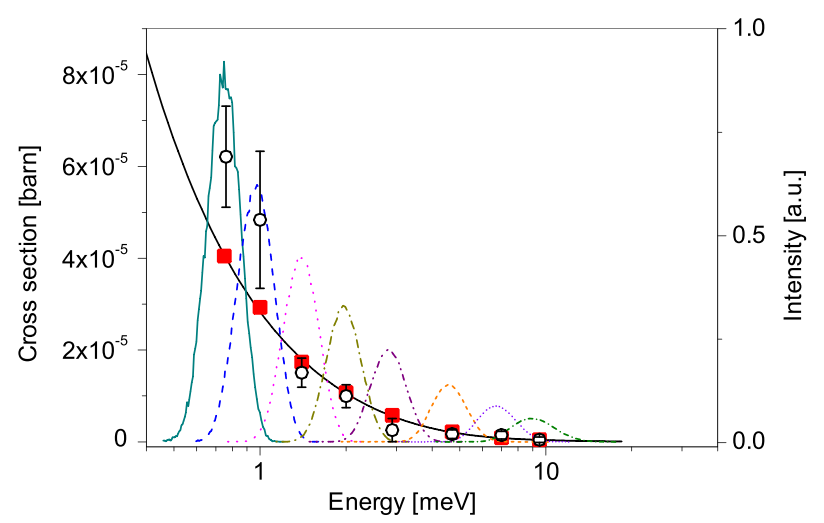

FIG. 3 (color online). Scaled measured (open circles) and calculated UCN production cross sections per molecule (Young and Koppel model: continuous black line and red squares; see text) for gaseous ortho ${ }^{2} \mathrm{H}_{2}$ at $0.12 \mathrm{MPa}$ and $25 \mathrm{~K}$. The velocity-selected $\mathrm{CN}$ intensity distributions are normalized to the same intensity. 


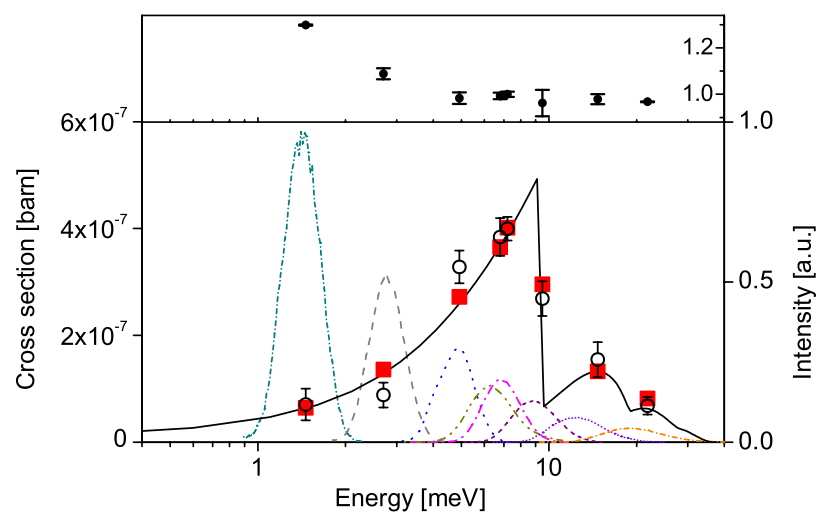

FIG. 4 (color online). Scaled measured (open circles) and calculated UCN production cross sections per molecule (multiphonon Debye model: continuous black line and red squares; see text) for solid ortho ${ }^{2} \mathrm{H}_{2}$ at $8 \mathrm{~K}$. The velocity-selected $\mathrm{CN}$ intensity distributions are normalized to the same intensity. The inset at the top displays the calculated energy dependent correction factors for the $\mathrm{CN}$ energy bins.

velocity component, there will always be more molecules available to downscatter $\mathrm{CN}$ of lower energy.

The fit of the data of solid ${ }^{2} \mathrm{H}_{2}$ to the simple Debye model results in $\chi^{2}=9.73$ for 7 degrees of freedom $\left(\chi_{\text {red }}^{2}=1.39\right)$. Qualitatively one again understands that the energy dependence follows the final state density of the phonons in the solid. Interestingly, the fit gets considerably worse when using the "more realistic" phonon density of states of $[11,25]$. It is, however, difficult to draw final conclusions from this discrepancy because of some systematic issues that are difficult to estimate, particularly differences between the crystals in the Bragg region (see Fig. 2) and also the validity of the incoherent approximation for Eq. (1). Nevertheless, we would like to emphasize the good agreement of the data and the Debye model in Fig. 4. In particular, the UCN production above $10 \mathrm{meV}$ provides strong evidence for two- and threephonon contributions [31].

The work was performed at the Swiss Spallation Neutron Source, PSI, Switzerland. We acknowledge the outstanding support of W. Arrigoni, M. Meier, and P. Schurter, and the help of K. Clausen, G. Frei, E. Lehmann, and T. Scherer with the velocity selector and imaging plate measurements as well of M. Luethy and M. Wiedemeier with the gold foil measurements. We thank E. Widmann, A. Wokaun, and J. Zmeskal for discussions.

*Also at Institute for Technical Chemistry, ETH Zürich, $\mathrm{CH}$.

${ }^{\dagger}$ Present address: Stanford University, Palo Alto, CA, USA.

${ }^{\ddagger}$ Also at Physik-Institut, University of Zürich, $\mathrm{CH}$.
${ }^{\S}$ Also at Stefan Meyer Institut für subatomare Physik, Austrian Academy of Sciences, Vienna, Austria. malgorzata.kasprzak@psi.ch

${ }^{\|}$Corresponding author. klaus.kirch@psi.ch

${ }^{\mathbb{I}}$ Also at Paul Scherrer Institut, CH-5232 Villigen PSI.

[1] E. Fermi and W. N. Zinn, Phys. Rev. 70, 103 (1946).

[2] M. L. Goldberger and F. Seitz, Phys. Rev. 71, 294 (1947).

[3] E. Fermi and L. Marshall, Phys. Rev. 71, 666 (1947).

[4] E. Fermi, Ricerca Scientifica 7, 13 (1936).

[5] V.K. Ignatovich, The Physics of Ultracold Neutrons (Clarendon Press, Oxford, 1990);

[6] R. Golub, D. J. Richardson, and S. K. Lamoreaux, Ultracold Neutrons (Adam Hilger, Bristol, 1991).

[7] A solid ${ }^{2} \mathrm{H}_{2} \mathrm{UCN}$ source is under construction at PSI; see F. Atchison et al., "The UCN Source at PSI," in Proceedings of the International Collaboration on Advanced Neutron Sources, ICANS-XVIII, Dongguan, China, 2007; see http://www.icans-Xviii.ac.cn/. Other efforts are under way at Los Alamos National Laboratory, ILL Grenoble, RCNP Osaka, Mainz University, TU Munich, and NC State University.

[8] R. Golub and J.M. Pendlebury, Phys. Lett. 62A, 337 (1977).

[9] I. S. Altarev et al., Phys. Lett. 80A, 413 (1980).

[10] R. Golub and K. Böning, Z. Phys. B 51, 95 (1983).

[11] Z.-Ch. Yu, S. S. Malik, and R. Golub, Z. Phys. B 62, 137 (1986).

[12] A. Serebrov et al., Nucl. Instrum. Methods Phys. Res., Sect. A 440, 658 (2000).

[13] C. L. Morris et al., Phys. Rev. Lett. 89, 272501 (2002).

[14] A. Saunders et al., Phys. Lett. B 593, 55 (2004).

[15] C.-Y. Liu, A.R. Young, and S.K. Lamoreaux, Phys. Rev. B 62, R3581 (2000).

[16] F. Atchison et al., Phys. Rev. Lett. 94, 212502 (2005).

[17] F. Atchison et al., Phys. Rev. Lett. 95, 182502 (2005).

[18] F. Atchison et al., Phys. Rev. C 71, 054601 (2005).

[19] J.A. Young and J. U. Koppel, Phys. Rev. 135, A603 (1964).

[20] J. U. Koppel and J. A. Young, Nukleonika 8, 40 (1966).

[21] G. C. Wick, Phys. Z. 38, 403 (1937).

[22] V. F. Turchin, Slow Neutrons (Israel Program for Scientific Translations, Jerusalem, 1965).

[23] S.W. Lovesey, Theory of Neutron Scattering from Condensed Matter (Clarendon Press, Oxford, 1984).

[24] A. P. Serebrov et al., JETP Lett. 59, 757 (1994).

[25] M. Nielsen and H. B. Moller, Phys. Rev. B 3, 4383 (1971).

[26] I. F. Silvera, Rev. Mod. Phys. 52, 393 (1980).

[27] We consider (and calculate the production of) UCN with [0..250] neV energy at $1 \mathrm{~m}$ height, corresponding to about [100...350] neV behind the production target and inside the gas target, but [0..250] neV inside solid ${ }^{2} \mathrm{H}_{2}$.

[28] J. Zejma et al., Nucl. Instrum. Methods Phys. Res., Sect. A 539, 622 (2005).

[29] K. Bodek et al., Nucl. Instrum. Methods Phys. Res., Sect. A 533, 491 (2004).

[30] I. Heilmann and J. Kjems, Ris $\phi-M-2208$ (Ris $\varnothing$ National Laboratory, Denmark, 1980).

[31] M. Kasprzak, Doctoral thesis, University of Vienna. 